\title{
Enlarged Left Atrium And Decreased Left Atrial Strain are Associated with Atrial Fibrillation in Patients with Hyperthyroidism Irrespective of Conventional Risk Factors
}

\author{
Dong Geum Shin \\ Kangnam Sacred Heart Hospital \\ Min-Kyung Kang ( $\sim$ homes78@naver.com ) \\ Kangnam Sacred Heart Hospital \\ Donghoon Han \\ Kangnam Sacred Heart Hospital \\ Seonghoon Choi \\ Kangnam Sacred Heart Hospital \\ Jung Rae Cho \\ Kangnam Sacred Heart Hospital \\ Namho Lee \\ Kangnam Sacred Heart Hospital
}

\section{Research Article}

Keywords: hyperthyroidism, atrial fibrillation, left atrium

Posted Date: August 19th, 2021

DOl: https://doi.org/10.21203/rs.3.rs-817961/v1

License: (c) (1) This work is licensed under a Creative Commons Attribution 4.0 International License.

Read Full License

Version of Record: A version of this preprint was published at The International Journal of Cardiovascular Imaging on October 27th, 2021. See the published version at https://doi.org/10.1007/s10554-021-024506. 


\section{Abstract}

Background: The purpose of this study was to investigate factors associated with AF in patients with hyperthyroidism beyond heart failure (HF), coronary heart disease (CHD), or valvular diseases.

Methods: A total of 136 patients (mean age, $52 \pm 15$ years; 86 [63\%] female) who were diagnosed with hyperthyroidism for the first time were enrolled. Patients who had HF, CHD, or significant valvular diseases were excluded. Patients were classified into two groups according to the presence (group 1 , $n=40$ ) and absence of AF (group 2, n=96).

Results: AF occurred in 40 (29\%) patients and $23(58 \%)$ of these patients showed paroxysmal AF. Among the symptoms of hyperthyroidism, the most common chief complaint was palpitation (30\%). Advanced age, presence of prior cerebrovascular events, and presence of palpitations were associated with AF. Larger left atrial volume index (LAVI), increased left ventricular mass index, and decreased left ventricular ejection fraction (LVEF) and S' velocity were associated with AF. Among them, presence of palpitations and increased LAVI were independently associated with the occurrence of AF. In addition, strain analysis, decreased LA expansion index (EI), ejection fraction (EF), peak atrial longitudinal strain, contraction strain, and late diastolic strain rate (A sr) and systolic strain rate ( $\mathrm{S} \mathrm{sr}$ ) were associated with the occurrence of AF and LAVI.

Conclusion: Presence of palpitations and enlarged left atrium were associated with the occurrence of AF in patients with hyperthyroidism irrespective of conventional risk factors. Additional LA analysis revealed that decreased LA function was associated with AF and enlarged left atrium.

\section{Introduction}

Atrial fibrillation (AF) is one of the most common cardiac arrhythmias and its incidence increases with age [1]. AF can occur in various cardiac and medical conditions, including hypertension, coronary heart disease (CHD), heart failure (HF), valvular heart disease, obesity [2,3], and sleep-apnea syndrome [4]. In addition to age and other conditions, it is well known that high consumption of alcohol can increase the probability of developing AF [5]. There are also a variety of acute conditions or potentially reversible triggers that can initiate AF, such as cardiac surgery [6], lung diseases [7], etc. Among them, hyperthyroidism is also known to be associated with the occurrence of AF $[8,9]$. Patients with hyperthyroidism have more premature supraventricular depolarizations, premature atrial beats, and nonsustained supraventricular tachycardia; increased heart rate; and reduced heart rate variability even without overt heart problems [10]. The electrical triggers caused due to the above-mentioned conditions may contribute to paroxysmal atrial tachycardia, $A F$, and atrial flutter. Among these arrhythmias, $A F$ is the most common and occurs in $5-15 \%$ of patients especially patients $\geq 60$ years of age [11]. Factors associated with increased risk include male sex, increasing age, HF, CHD, and valvular heart disease [9]. As mentioned previously, AF-irrespective of thyroid function-is believed to be caused due to chaotic electrical activity resulting in micro-reentrant tachycardia [12]. One of the other possible mechanisms of 
$\mathrm{AF}$ is the presence of anatomical substrate and abnormal ectopic atrial firing. In a hyperthyroidic state, elevated thyroid hormone alters the B1-adrenergic and M2-muscarinic receptors of the heart, resulting in increased sympathetic function, tachycardia, decreased atrial refractory period, and altered ionic channels [13]. AF in human patients with hyperthyroidism, similar to the animal models, is believed to be due to a decreased atrial refractory period and increased sympathetic tone with decreased heart rate variability [14]. Complications of AF in patients with hyperthyroidism include HF and thromboembolism, although it remains controversial whether $A F$ in hyperthyroidism is associated with a higher thromboembolic risk than that of AF in other conditions [15]. Therefore, it is not clear whether AF occurs in hyperthyroidism due to patient-associated risk factors-such as HF, CHD, or significant valvular disease -or a thyroid problem. Therefore, the purpose of this study was to determine the clinical characteristics of patients with AF in patients diagnosed with hyperthyroidism for the first time without HF, CHD, or significant valvular diseases.

\section{Methods}

\section{Study design and participants}

This was a retrospective study of 136 patients who were diagnosed with hyperthyroidism for the first time and were not using thyroid-related drugs (86 [63\%] female, mean age: $52 \pm 15$ years). Patients attended the Kangnam Sacred Heart Hospital, Hallym University between July 2009 and February 2021. We excluded the following patients: those in a euthyroid state and taking drugs for hyperthyroidism, with hyperthyroidism and taking drugs for hypothyroidism, who did not undergo echocardiography, and with a left ventricular ejection fraction (LVEF) $<50 \%$, preexisting $\mathrm{HF}$, known $\mathrm{CHD}$, acute coronary syndrome, or significant valvular dysfunction.

Patients were classified into two categories according to the presence or absence of AF [group 1: with AF, $\mathrm{n}=40(29 \%)$ vs. group 2: without AF, n=96 (71\%)]. Seventeen patients had chronic AF and $23 \mathrm{had}$ paroxysmal AF (PAF). Clinical, laboratory, and echocardiographic parameters were compared between the two groups.

All procedures performed were in accordance with the ethical standards of the Institutional Research Committee and the 1964 Declaration of Helsinki and its later amendments. The need for informed consent was waived because of the retrospective design of this study. The authors had no access to information that could potentially identify patients.

\section{Echocardiography and left atrial strain analysis}

Transthoracic echocardiography (TTE) was performed using standard techniques with a 2.5-MHz transducer. TTE was performed by a well-trained sonographer (with an experience of over 6 months) and results were confirmed by a cardiologist almost in real time. Standard 2-dimensional (2-D) and Doppler 
echocardiography were performed using a commercially available echocardiographic machine (Vivid 7R; GE Medical System, Horten, Norway) having the same setup and interfaced with a 2.5-MHz phased-array probe. All measurements were performed according to the guidelines [16]. With the study participant in the partial left decubitus position and breathing normally, the observer obtained images from the parasternal long and short axes and from the apical four- and two-chamber and long-axis views. Depth setting was optimized to display the left ventricle as large as possible on the screen and the same field depth was kept for the four- and two-chamber apical views. Sector width was reduced to increase spatial and temporal resolution. The left ventricular end-diastolic dimensions (LVEDD), end-diastolic interventricular septal thickness, and end-diastolic left ventricular (LV) posterior wall thickness were measured at end-diastole according to the standards established by the American Society of Echocardiography. The LV EF was determined using the biplane Simpson's method. The maximal left atrial (LA) volume was calculated using the Simpson method and indexed to the body surface area. The LV mass was calculated using the Devereux formula as 1.04 [(LVEDD + IVSTd + PWTd $\left.)^{3}-(\text { LVEDD })^{3}\right]-$ 13.6. Thereafter, the left ventricular mass index (LVMI) was calculated and indexed to body surface area.

Mitral flow velocities were recorded in the apical four-chamber view. Mitral inflow measurements included the peak early $(E)$ and peak late $(A)$ flow velocities and the E/A ratio. The tissue Doppler of the mitral annulus movement was also obtained from the apical four-chamber view. A 1.5-mm sample volume was placed sequentially at the septal annular sites. The analysis was performed for early diastolic ( $\left.E^{\prime}\right)$, late diastolic ( $\left.A^{\prime}\right)$ and systolic $\left(S^{\prime}\right)$ peak tissue velocities. As a noninvasive parameter for the LV stiffness, the LV filling index ( $\left.E / E^{\prime}\right)$ was calculated by the ratio of trans-mitral flow velocity to annular velocity. Adequate mitral and tissue Doppler image signals were recorded in all patients.

\section{Additionnal left atrial volumetric and strain analysis}

In addition, the LA expansion index (EI) was calculated as $\left(\mathrm{Vol}_{\mathrm{max}}-\mathrm{Vol}_{\mathrm{min}}\right) \times 100 \% / \mathrm{Vol}_{\mathrm{min}}$. For patients with $A F$, the LA El was calculated as the average of measurements in five consecutive beats. In all patients, the LA volumes were indexed to the body surface area [17]. LA EF was also calculated as $\left(\mathrm{Vol}_{\max }-\mathrm{Vol}_{\min }\right) / \mathrm{Vol}_{\max }[18]$. The LA strain measurements were obtained by 2-D speckle tracking echocardiography (STE) [19]. The peak atrial longitudinal strain (PALS) and peak atrial contraction strain (PACS) and the LA strain rate (SR) in ventricular systole (S sr), early diastole (E sr), and late diastole (A sr) were measured [19].

\section{Statistical analysis}

All continuous data were expressed as mean \pm standard deviation, and all categorical data are presented as percentages or absolute numbers. Continuous variables were analyzed using one-way analysis of variance in three independent groups and Student's t-test in two independent groups, and dichotomous variables were analyzed using the chi-square test. Cox regression analysis was performed to evaluate significant variables associated with AF. The predictive abilities of the LAVI and LA EI were determined by the area under the receiver-operating characteristic (ROC) curves. The correlation between LAVI and other 
functional parameters, including LA strain and SR, was investigated using the Pearson correlation analysis. A P-value of less than 0.05 was considered significant. All tests were performed using Statistical Package for the Social Sciences version 27.0 (SPSS Inc., Chicago, IL, USA).

\section{Results}

Comparison of baseline characteristics and chief complaints associated with hyperthyroidism (Table 1)

Table 1.. Baseline characteristics of the study population

\begin{tabular}{llll} 
& $\mathrm{AF}(\mathrm{n}=40)$ & No AF $(\mathrm{n}=96)$ & $\mathrm{p}$ \\
\hline Age (years) & $59 \pm 15$ & $49 \pm 14$ & $<0.001$ \\
\hline male gender & $18(45 \%)$ & $32(33 \%)$ & 0.242 \\
\hline SBP $(\mathrm{mmHg})$ & $119 \pm 16$ & $121 \pm 15$ & 0.477 \\
\hline DBP & $70 \pm 11$ & $73 \pm 10$ & 0.094 \\
\hline Heart rate $(\mathrm{bpm})$ & $84 \pm 19$ & $84 \pm 20$ & 0.958 \\
\hline Body mass index $\left(\mathrm{kg} / \mathrm{m}^{2}\right)$ & $23.7 \pm 4.0$ & $23.5 \pm 3.6$ & 0.815 \\
\hline DM & $6(15 \%)$ & $9(9 \%)$ & 0.374 \\
\hline Hypertension & $6(15 \%)$ & $23(24 \%)$ & 0.358 \\
\hline Alcohol & $12(30 \%)$ & $16(17 \%)$ & 0.103 \\
\hline smoking & $8(20 \%)$ & $13(14 \%)$ & 0.435 \\
\hline CVA & $6(15 \%)$ & $2(2 \%)$ & 0.008 \\
\hline Chief complaints & & & 0.120 \\
\hline palpitation & $18(45 \%)$ & $23(24 \%)$ & \\
\hline Chest pain, DOE & $2(5 \%)$ & $20(21 \%)$ & \\
\hline dizziness & $3(8 \%)$ & $7(7 \%)$ & \\
\hline weakness & $6(15 \%)$ & $17(18 \%)$ & \\
\hline Presence of palpitation & $19(48 \%)$ & $26(27 \%)$ & 0.028 \\
\hline
\end{tabular}

SBP: systolic blood pressure, DBP: diastolic BP, DM: diabetes mellitus, CVA: cerebrovascular attack, DOE: dyspnea on exertion

AF was diagnosed in $40(29 \%)$ of 136 patients, and among these patients, 23 (58\%) were diagnosed with PAF. The most common symptom of hyperthyroidism was palpitations. 
Patients with AF were compared to those without (59 \pm 15 vs. $49 \pm 14$ years, $p<0.001$ ), and cerebrovascular attack (CVA) was more prevalent in patients with $A F$ than in those without $(15 \% \mathrm{vs} .2 \%$, $\mathrm{p}=0.008$ ). Although palpitations were the most common symptom in both the groups, palpitations were more common in patients with $\mathrm{AF}$ than in those without ( $48 \%$ vs. $27 \%, p=0.028)$.

Echocardiographic and laboratory findings of the study population (Table 2)

Table 2. Echocardiographic and laboratory parameters of study population

\begin{tabular}{|c|c|c|c|}
\hline & $\mathrm{AF}(\mathrm{n}=40)$ & No AF $(n=96)$ & $\mathrm{p}$ \\
\hline LAVI $\left(\mathrm{ml} / \mathrm{m}^{2}\right)$ & $36.6 \pm 14.9$ & $26.0 \pm 7.9$ & $<0.001$ \\
\hline LV EDD (mm) & $49.3 \pm 5.3$ & $48.5 \pm 4.7$ & 0.381 \\
\hline LV ESD & $31.9 \pm 6.8$ & $30.6 \pm 4.8$ & 0.218 \\
\hline LVMI $\left(\mathrm{g} / \mathrm{m}^{2}\right)$ & $98.6 \pm 23.0$ & $86.4 \pm 18.7$ & 0.002 \\
\hline LV EF (\%) & $61.9 \pm 5.9$ & $66.7 \pm 7.1$ & $<0.001$ \\
\hline $\mathrm{E}(\mathrm{cm} / \mathrm{s})$ & $82.0 \pm 24.0$ & $80.9 \pm 21.1$ & 0.785 \\
\hline $\mathrm{A}(\mathrm{cm} / \mathrm{s})$ & $68.2 \pm 20.3$ & $73.1 \pm 20.1$ & 0.298 \\
\hline E/A ratio & $1.09 \pm 0.40$ & $1.19 \pm 0.48$ & 0.380 \\
\hline DT (ms) & $182.9 \pm 48.1$ & $190.5 \pm 37.0$ & 0.331 \\
\hline $\mathrm{E}^{\prime}(\mathrm{cm} / \mathrm{s})$ & $9.0 \pm 3.1$ & $9.2 \pm 2.7$ & 0.639 \\
\hline$A^{\prime}(\mathrm{cm} / \mathrm{s})$ & $8.9 \pm 1.5$ & $9.5 \pm 2.3$ & 0.289 \\
\hline$E^{\prime} / A^{\prime}$ & $0.88 \pm 0.34$ & $1.04 \pm 0.43$ & 0.105 \\
\hline$E / E^{\prime}$ & $10.1 \pm 3.8$ & $9.3 \pm 3.0$ & 0.220 \\
\hline $\mathrm{S}^{\prime}(\mathrm{cm} / \mathrm{s})$ & $7.5 \pm 1.8$ & $9.1 \pm 2.5$ & $<0.001$ \\
\hline RVSP & $35.4 \pm 9.6$ & $33.0 \pm 9.2$ & 0.190 \\
\hline TSH (ulU/mL) & $0.013 \pm 0.013$ & $0.010 \pm 0.014$ & 0.188 \\
\hline FT4 (ng/dL) & $3.59 \pm 2.86$ & $4.00 \pm 2.61$ & 0.454 \\
\hline T3 (ng/dL) & $308.0 \pm 215.0$ & $343.3 \pm 207.9$ & 0.378 \\
\hline $\mathrm{BNP}(\mathrm{pg} / \mathrm{mL})$ & $191.6 \pm 138.1$ & $115.1 \pm 150.6$ & 0.060 \\
\hline CK-MB (ng/mL) & $2.63 \pm 3.45$ & $2.31 \pm 5.79$ & 0.813 \\
\hline $\mathrm{Tnl}(\mathrm{ng} / \mathrm{mL})$ & $1.60 \pm 6.65$ & $0.09 \pm 0.29$ & 0.277 \\
\hline
\end{tabular}


LAVI: left atrial volume index, LV: left ventricular, EDD: end-diastolic dimension, ESD: end-systolic dimension, LVMI: LV mass index, EF: ejection fraction, RVSP: right ventricular systolic pressure, TSH: thyroid stimulating hormone, FT4: free T 4, BNP: brain natriuretic peptide, CK-MB: creatinine kinasemusclebrain, Tnl: troponin I.

The LAVI was significantly larger $(37 \pm 15 \mathrm{~mL} / \mathrm{m} 2$ vs. $26 \pm 8 \mathrm{~mL} / \mathrm{m} 2, \mathrm{p}<0.001)$ in patients with AF than in those without. Although within the normal range, the LVMI was larger $(99 \pm 23 \mathrm{~g} / \mathrm{m} 2 \mathrm{vs.} 86 \pm 19 \mathrm{~g} / \mathrm{m} 2$, $\mathrm{p}=0.002)$ and the LVEF was lower $(62 \pm 6 \%$ vs. $67 \pm 7 \%, p<0.001)$ in patients with AF. Other diastolic parameters, including $E / E^{\prime}$, did not differ significantly between the two groups, but $\mathrm{S}^{\prime}$ velocity was lower $(7.5 \pm 1.8 \mathrm{~cm} / \mathrm{s}$ vs. $9.1 \pm 2.5 \mathrm{~cm} / \mathrm{s}, \mathrm{p}<0.001)$ in patients with AF. There was no significant difference between the two groups for blood test results of thyroid function and cardiac biomarkers, but the brain natriuretic peptide level was slightly higher in patients with $\mathrm{AF}$ with borderline significance than in those without $(192 \pm 138 \mathrm{pg} / \mathrm{mL}$ vs. $115 \pm 151 \mathrm{pg} / \mathrm{mL}, \mathrm{p}=0.060)$.

Independent predictors of atrial fibrillation in patients with hyperthyroidism (Table 3)

Table 3. Independent predictors for occurrence of atrial fibrillation in patients with hyperthyroidism

\begin{tabular}{|llll|}
\hline & OR & $95 \% \mathrm{Cl}$ & $\mathrm{P}$ \\
\hline Univariate & & & \\
\hline age & 1.052 & $1.022-1.084$ & 0.001 \\
\hline CVA & 8.294 & $1.597-43.087$ & 0.012 \\
\hline palpitation & 2.436 & $1.132-5.243$ & 0.023 \\
\hline LAVI & 1.101 & $1.052-1.153$ & $<0.001$ \\
\hline LVMI & 1.030 & $1.010-1.051$ & 0.003 \\
\hline EF & 0.902 & $0.848-0.960$ & 0.001 \\
\hline S' & 0.699 & $0.570-0.857$ & 0.001 \\
\hline Multivariate & & & \\
\hline age & 1.017 & $0.979-1.057$ & 0.390 \\
\hline CVA & 4.087 & $0.493-33.872$ & 0.192 \\
\hline palpitation & 5.636 & $1.987-15.986$ & 0.001 \\
\hline LAVI & 1.092 & $1.030-1.159$ & 0.003 \\
\hline LVMI & 1.010 & $0.982-1.038$ & 0.480 \\
\hline LV EF & 0.967 & $0.890-1.051$ & 0.428 \\
\hline LV S' & 0.834 & $0.649-1.072$ & 0.157 \\
\hline
\end{tabular}


CVA: cerebrovascular attack, LAVI: left atrial volume index, LVMI: left ventricular mass index, EF: ejection graction. S': S' velocity.

Younger age, CVA, presence of palpitations, larger LAVI, higher LVMI, and lower EF and S' velocity were found to be associated with $A F$ in the univariate analysis. Among them, presence of palpitations (odds ratio [OR]: 5.636, 95\% confidence interval [Cl]: 1.987-15.986, $p=0.001)$ and larger LAVI (OR: $1.092,95 \% \mathrm{Cl}$ : $1.030-1.159, p=0.003$ ) were found to be independently associated with the occurrence of $A F$ in patients with hyperthyroidism.

Left atrial expansion index, ejection fraction, and strain analysis (Table 4)

Table 4. LA El, EF and strain, strain rate and other parameters of the study population

\begin{tabular}{|c|c|c|c|c|}
\hline & $\mathrm{AF}(\mathrm{n}=40)$ & No $A F(n=96)$ & & $\mathrm{p}$ \\
\hline LA El (\%) & $74.5 \pm 46.9$ & $126.6 \pm 69.4$ & & 0.001 \\
\hline LA EF (\%) & $49.8 \pm 16.0$ & $63.3 \pm 10.6$ & & $<0.001$ \\
\hline PALS & $19.3 \pm 9.9$ & $27.6 \pm 9.5$ & & $<0.001$ \\
\hline PACS ${ }^{*}$ & $9.0 \pm 5.4$ & $12.8 \pm 4.1$ & & 0.002 \\
\hline LA E SR & $-1.07 \pm 0.61$ & $-1.31 \pm 0.59$ & & 0.085 \\
\hline LA A SR ${ }^{*}$ & $-1.10 \pm 0.59$ & $-1.67 \pm 0.55$ & & $<0.001$ \\
\hline LA S SR & $0.95 \pm 0.46$ & $1.37 \pm 0.45$ & & $<0.001$ \\
\hline \multicolumn{5}{|c|}{ Correlation between LAVI and LA strain and strain rate } \\
\hline Variables & \multicolumn{2}{|c|}{ Pearson correlation } & $\mathrm{p}$ & \\
\hline LAEI (\%) & \multicolumn{2}{|l|}{-0.338} & \multicolumn{2}{|l|}{0.002} \\
\hline LA EF (\%) & \multicolumn{2}{|l|}{-0.481} & \multicolumn{2}{|l|}{$<0.001$} \\
\hline PALS & \multicolumn{2}{|l|}{-0.320} & \multicolumn{2}{|l|}{0.004} \\
\hline PACS & \multicolumn{2}{|l|}{-0.416} & \multicolumn{2}{|l|}{$<0.001$} \\
\hline LA A sr & \multicolumn{2}{|l|}{-0.407} & \multicolumn{2}{|l|}{$<0.001$} \\
\hline LAS sr & \multicolumn{2}{|l|}{-0.367} & \multicolumn{2}{|l|}{0.001} \\
\hline
\end{tabular}

LA: left atrial, R:reservoir function, LA EF: LA ejection fraction. LAEI : LA expansion index, PALS: peak atrial longitudinal strain, PACS: peak atrial contraction strain, LA E, A and S sr: LA E, A and S strain rate, *: in patients with PAF (sinus rhythm during echocardiography) 
Table 4 shows the additional LA volumetric and strain analysis. The LA El (75 $\pm 47 \%$ vs. $127 \pm 69 \%$, $\mathrm{p}<0.001)$ and LA EF (75 $\pm 47 \%$ vs. $127 \pm 69 \%, p<0.001)$ were significantly decreased in patients with AF than in those without. Figure 1 shows the ROC curve for LAVI (cut-off value $\geq 24 \mathrm{~mL} / \mathrm{m} 2$, area under the curve [AUC]: 0.769, SE: 0.055, p<0.001), LA El (cut-off value $\leq 81 \%$, AUC: 0.224, SE: 0.056, p<0.001), and LA EF (cut-off value $\leq 56 \%$, AUC: 0.224 , SE: 0.058 , p<0.001). In the strain analysis, both PALS and PACS were decreased in patients with AF. The strain rate analysis showed that LA A sr and S sr were decreased in patients with AF. The LA El, EF, PALS, PACS, A sr, and S sr all showed negative correlations with the LAVI. Figure 2 shows each of these correlations.

\section{Discussion}

In this study, AF occurred in 40 (29\%) patients and 23 (58\%) patients showed PAF. Among the symptoms of hyperthyroidism, the most common complaint was palpitations (30\%) and 33\% reported experiencing palpitations. (Supplementary Table). Advanced age, presence of prior cerebrovascular events, and presence of palpitations were associated with AF. In echocardiographic parameters, larger LAVI, increased LVMI, and decreased LVEF and S' velocity were associated with AF. Among these parameters, the presence of palpitations and increased LAVI were independently associated with the occurrence of AF. In additionnal LA volumetric and strain analyses, decreased LA EI, EF, PALS, contraction strain, and A sr and $\mathrm{S}$ sr were associated with the occurrence of AF and higher LAVI.

\section{Occurrence of AF in this study irrespective of conventional risk factors}

In one population-based study of 40,628 patients with clinical hyperthyroidism, $8.3 \%$ had AF or atrial flutter [20]. Another retrospective cross-sectional study compared AF prevalence in 1338 subjects with overt or subclinical hyperthyroidism. According to the study, the prevalence of AF was $13.8 \%$ in patients with overt hyperthyroidism, $12.7 \%$ in those with subclinical hyperthyroidism, and $2.3 \%$ in euthyroid controls [21]. Compared with these studies, the prevalence of AF was higher (29\%) in this study. However, by comparing the results, it cannot be said that the prevalence of AF was found to be high or the frequency of AF occurrence was found to be increased in this study. This may be because this study was conducted only in patients who were diagnosed with hyperthyroidism for the first time and had undergone echocardiography and electrocardiography. Therefore, since there were patients who did not undergo echocardiography or whose results could not be confirmed despite experiencing symptoms, such as palpitations and angina-like symptoms, the high frequency of AF seen in this study may be attributed to a possible selection bias. However, the high frequency of AF seen in this study is an important finding as AF was diagnosed in a large number of patients even though patients with conventional risk factors$\mathrm{HF}, \mathrm{CHD}$, or significant valvular disease for AF-were excluded.

Factors associated with increased risk of AF in patients with hyperthyroidism include male sex, increasing age, HF, CHD, and valvular heart disease [9]. The association of AF with increasing age presumably reflects the age-related reduction in the threshold for developing AF [1]. In this study, although 
patients with AF were older ( $59 \pm 15$ vs. $49 \pm 14$ years), advanced age was not independently associated with the occurrence of AF. This may be due to the inclusion of a relatively young age group of patients with hyperthyroidism in this study. Moreover, it is known that AF is rare in healthy young adults [22], and the average age of patients included in this study was $52 \pm 15$ years. Additionally, patients who were diagnosed with hyperthyroidism for the first time were included in this study and those with decreased LV systolic function ( $E F<50 \%$ ), previously diagnosed $\mathrm{HF}, \mathrm{CHD}$, and significant valve disease were excluded. Therefore, it is considered that relatively young patients were enrolled in this study.

\section{Association between palpitations and occurrence of AF}

Palpitations, exercise intolerance, dyspnea, angina-like chest pain, peripheral edema, and congestive HF are common symptoms of hyperthyroidism $[23,24]$. Cardiovascular effects of hyperthyroidism are the most common and dangerous effects causing patients to seek treatment in hospitals [25]. In this study, palpitations and angina-like chest pain or dyspnea were the most common symptoms followed by dizziness, general weakness, and weight loss. Even though sinus tachycardia is the most common rhythm disturbance in patients with hyperthyroidism, its clinical importance is less than that of AF, which occurs in $5-15 \%$ of patients with hyperthyroidism, which may be the presenting problem [26]. The higher prevalence rates have been found in studies involving older patients with known or suspected underlying organic heart disease [27]. A large study found that less than $1 \%$ new-onset AF was caused by overt hyperthyroidism [15]. Therefore, although it is recommended to measure serum thyrotropin in all patients with new-onset AF to rule out thyroid disease, this association is uncommon in the absence of additional signs and symptoms of hyperthyroidism [28]. Similarly, in this study, there were significantly more patients with palpitations in the AF group. Therefore, it is necessary to make efforts in diagnosing AF in patients with hyperthyroidism complaining of palpitations.

\section{Enlarged left atrial and decreased left atrial strain vs. occurrence of atrial fibrillation}

It is well known that an increased LA size is associated with the occurrence of AF $[29,30]$. The left atrium is an important chamber of the heart that collects blood into the heart and modulates blood flow into the left ventricle during systole and diastole, respectively [31]. An enlarged left atrium is the hallmark of the structural remodeling process, which occurs in response to chronic pressure and volume overload. An enlarged left atrium most commonly occurs in association with diastolic dysfunction, LV hypertrophy, mitral valvular disease, and systemic hypertension [32]. It is well known that an enlarged left atrium can independently predict the development of clinically significant cardiovascular diseases and HF [33,34]. In the absence of mitral valvular disease, AF, and high cardiac output, it is an excellent indicator of LV diastolic dysfunction. In our study, patients with AF showed more enlarged left atrium (increased LAVI), and an enlarged LA was independently associated with the occurrence of AF.

In addition, decreased LA function measured by additional volumetric and strain analysis was associated with the occurrence of AF. Furthermore, decreased LA function was inversely associated with an enlarged left atrium. There are several parameters that define the LA function. Trans-mitral peak A velocity and tissue Doppler derived A' velocity are known to be suggestive of the contractile function of LA and well 
correlated with the volume of LA $[35,36]$. However, in this study, the LAVI was larger in patients with AF than in those without, but there was no significant difference in both trans-mitral A and tissue Doppler derived $A^{\prime}$ velocities between the two groups. However, using these measures mandates the presence of sinus rhythm, but $17(42 \%)$ patients with $A F$ had chronic $A F$, and therefore it was impossible to measure mitral inflow $A$ and tissue Doppler $A^{\prime}$ velocities in those patients. On the other hand, volumetric measures, including the LA EF and LA EI, have been utilized in both sinsu rhythm and AF $[17,18]$. Both LA EF and LA El were decreased in patients with AF and inversely correlated with LAVI. Strain analysis has also been utilized for evaluation of the LA function. We measured PALS and PACS by 2-D STE and LA SR in S sr, E sr, and A sr. PALS was reduced in patients with AF, and PACS, which could be measured only in patients with $S R$, was also reduced in patients with $A F$ unlike $A$ and $A^{\prime}$ velocities. It is known that the LA strain measurements are more sensitive than volumetric measures [18], and recent studies using 2-D STE have shown an association between reduced LA reservoir, contractile function, and PAF that precedes LA enlargement [36]. In addition, the LA A SR and S SR were decreased in patients with AF and inversely correlated with the LAVI. The results of this study indicate that although the LA strain and SR were less sensitive than the LA volume in reflecting the LA function, their values could reflect the LA function more sensitively than $A$ and $A^{\prime}$ velocities.

\section{Limitations}

This study had several limitations. First, this was a single center study with a relatively small study population. Second, since not all patients had undergone tests, such as the Holter or telemetry tests, the diagnosis of AF may have been missed. Third, this study was a retrospective study and did not follow the long-term prognosis of patients with AF. Finally, it was not possible to investigate whether AF occurred at follow-up after the diagnosis of hyperthyroidism during or after treatment. However, as this is the first study to investigate the prevalence of $A F$ and related factors in patients diagnosed with hyperthyroidism, we believe that these findings might help to better diagnose AF in patients with hyperthyroidism in actual clinical practice.

\section{Conclusion}

In conclusion, our findings highlight that presence of palpitations and enlarged left atrium were associated with the occurrence of AF in patients with hyperthyroidism irrespective of conventional risk factors. In additional analysis of LA volume and strain, decreased LA EI, LA EF, PALS, PACS, A sr and S sr a were associated with the occurrence of $A F$ and inversely related to enlarged left atrium.

\section{Declarations}

\section{Conflict of interests}

The authors have no conflicts of interest to declare. 


\section{References}

1. Wasmer K, Eckardt L, Breithardt G (2017) Predisposing factors for atrial fibrillation in the elderly. J Geriatr Cardiol 14:179-184

2. Staerk L, Sherer JA, Ko D, Benjamin EJ, Helm RH (2017) Atrial Fibrillation: Epidemiology, Pathophysiology, and Clinical Outcomes. Circ Res 120:1501-1517

3. Goudis CA, Korantzopoulos P, Ntalas IV, Kallergis EM, Ketikoglou DG (2015) Obesity and atrial fibrillation: A comprehensive review of the pathophysiological mechanisms and links. J Cardiol 66:361-369

4. Shapira-Daniels A, Mohanty S, Contreras-Valdes FM, Tieu H, Thomas RJ, Natale A, et al (2020) Prevalence of Undiagnosed Sleep Apnea in Patients With Atrial Fibrillation and its Impact on Therapy. JACC Clin Electrophysiol 6:1499-1506

5. Voskoboinik A, Prabhu S, Ling LH, Kalman JM, Kistler PM (2016) Alcohol and Atrial Fibrillation: A Sobering Review. J Am Coll Cardiol 68:2567-7256

6. Lubitz SA, Yin X, Rienstra M, Schnabel RB, Walkey AJ, Magnani JW, et al (2015) Long-term outcomes of secondary atrial fibrillation in the community: the Framingham Heart Study. Circulation 131:16481655

7. Vahdatpour CA, Luebbert JJ, Palevsky HI (2020) Atrial arrhythmias in chronic lung diseaseassociated pulmonary hypertension. Pulm Circ, 10:2045894020910685

8. Woeber KA (1992) Thyrotoxicosis and the heart. N Engl J Med 327:94-98

9. Reddy V, Taha W, Kundumadam S, Khan M (2017) Atrial fibrillation and hyperthyroidism: A literature review. Indian Heart J 69:545-550

10. Wustmann K, Kucera JP, Zanchi A, Burow A, Stuber T, Chappuis B, et al (2008) Activation of electrical triggers of atrial fibrillation in hyperthyroidism. J Clin Endocrinol Metab 93:2104-2108

11. Klein I, Danzi S (2007) Thyroid disease and the heart. Circulation 116:1725-1735

12. Markides V, Schilling RJ (2003) Atrial fibrillation: classification, pathophysiology, mechanisms and drug treatment. Heart 89:939-943

13. Watanabe H, Ma M, Washizuka T, Komura S, Yoshida T, Hosaka Y, et al (2003) Thyroid hormone regulates mRNA expression and currents of ion channels in rat atrium. Biochem Biophys Res Commun 308:439-444

14. Bielecka-Dabrowa A, Mikhailidis DP, Rysz J, Banach M (2009) The mechanisms of atrial fibrillation in hyperthyroidism. Thyroid Res 2:4

15. Klein I, Ojamaa K (2001) Thyroid hormone and the cardiovascular system. N Engl J Med 344:501509

16. Lang RM, Badano LP, Mor-Avi V, Afilalo J, Armstrong A, Ernande L, et al (2015) Recommendations for cardiac chamber quantification by echocardiography in adults: an update from the American Society of Echocardiography and the European Association of Cardiovascular Imaging. J Am Soc Echocardiogr 28:1-39 
17. Hsiao SH, Huang WC, Lin KL, Chiou KR, Kuo FY, Lin SK, et al (2010) Left atrial distensibility and left ventricular filling pressure in acute versus chronic severe mitral regurgitation. Am J Cardiol 105:709715

18. Hoit BD (2014) Left atrial size and function: role in prognosis. J Am Coll Cardiol 63:493-505.

19. Cameli M, Lisi M, Righini FM, Mondillo S (2012) Novel echocardiographic techniques to assess left atrial size, anatomy and function. Cardiovasc Ultrasound 10:4

20. Frost L, Vestergaard P, Mosekilde L (2004) Hyperthyroidism and risk of atrial fibrillation or flutter: a population-based study. Arch Intern Med 164:1675-1678

21. Auer J, Scheibner P, Mische T, Langsteger W, Eber O, Eber B (2001) Subclinical hyperthyroidism as a risk factor for atrial fibrillation. Am Heart J 142:838-842

22. HISS RG, LAMB LE (1962) Electrocardiographic findings in 122,043 individuals. Circulation 25:947965

23. Ghandour A, Reust C (2011) Hyperthyroidism: a stepwise approach to treatment. J Fam Pract 60: 388-395

24. Palitzsch KD (2008). Prevention and multimodal therapy of hyperthyroidism. Internist (Berl) 49: 1428-36

25. Ertek S, Cicero AF (2013) Hyperthyroidism and cardiovascular complications: a narrative review on the basis of pathophysiology. Arch Med Sci 9:944-952

26. Klein I, Ojamaa K (1998). Thyrotoxicosis and the heart. Endocrinol Metab Clin North Am 27:51-62

27. Nordyke RA, Gilbert FI Jr, Harada ASM (1988) Graves' disease: influence of age on clinical findings. Arch Intern Med 148:626-631

28. Krahn AD, Klein GJ, Kerr CR, Boone J, Sheldon R, Green M, et al (1996) How useful is thyroid function testing in patients with recent-onset atrial fibrillation? Arch Intern Med 156:2221-2224

29. Henry WL, Morganroth J, Pearlman AS, Clark CE, Redwood DR, Itscoitz SB, et al (1976) Relation between echocardiographically determined left atrial size and atrial fibrillation. Circulation 53:273279

30. Seko Y, Kato T, Haruna T, Izumi T, Miyamoto S, Nakane E, et al (2018) Association between atrial fibrillation, atrial enlargement, and left ventricular geometric remodeling. Sci Rep 8:6366

31. Abhayaratna WP, Seward JB, Appleton CP, Douglas PS, Oh JK, Tajik AJ, et al (2006) Left atrial size: physiologic determinants and clinical applications. J Am Coll Cardiol 47:2357-2363

32. Cuspidi C, Rescaldani M, Sala C (2013) Prevalence of echocardiographic left-atrial enlargement in hypertension: a systematic review of recent clinical studies. Am J Hypertens 26:456-464

33. Patel DA, Lavie CJ, Milani RV, Shah S, Gilliland Y (2009) Clinical implications of left atrial enlargement: a review. Ochsner J 9:191-196

34. Kizer JR, Bella JN, Palmieri V, Liu JE, Best LG, Lee ET, et al (2006) Left atrial diameter as an independent predictor of first clinical cardiovascular events in middle-aged and elderly adults: the Strong Heart Study (SHS). Am Heart J 151:412-418 
35. Manninh Wj, Silverman DI, Katz SE, Douglas PS (1993) Atrial ejection force: a noninvasive assessment of atrial systolic function. J Am Coll Cardiol 22:221-225

36. Hesse B, Schuele SU, Thamilasaran M, Thomas J, Rodriguez L (2004) A rapid method to quantify left atrial contractile function: Doppler tissue imaging of the mitral annulus during atrial systole. Eur $\mathrm{J}$ Echocardiogra 5:86-92

\section{Figures}

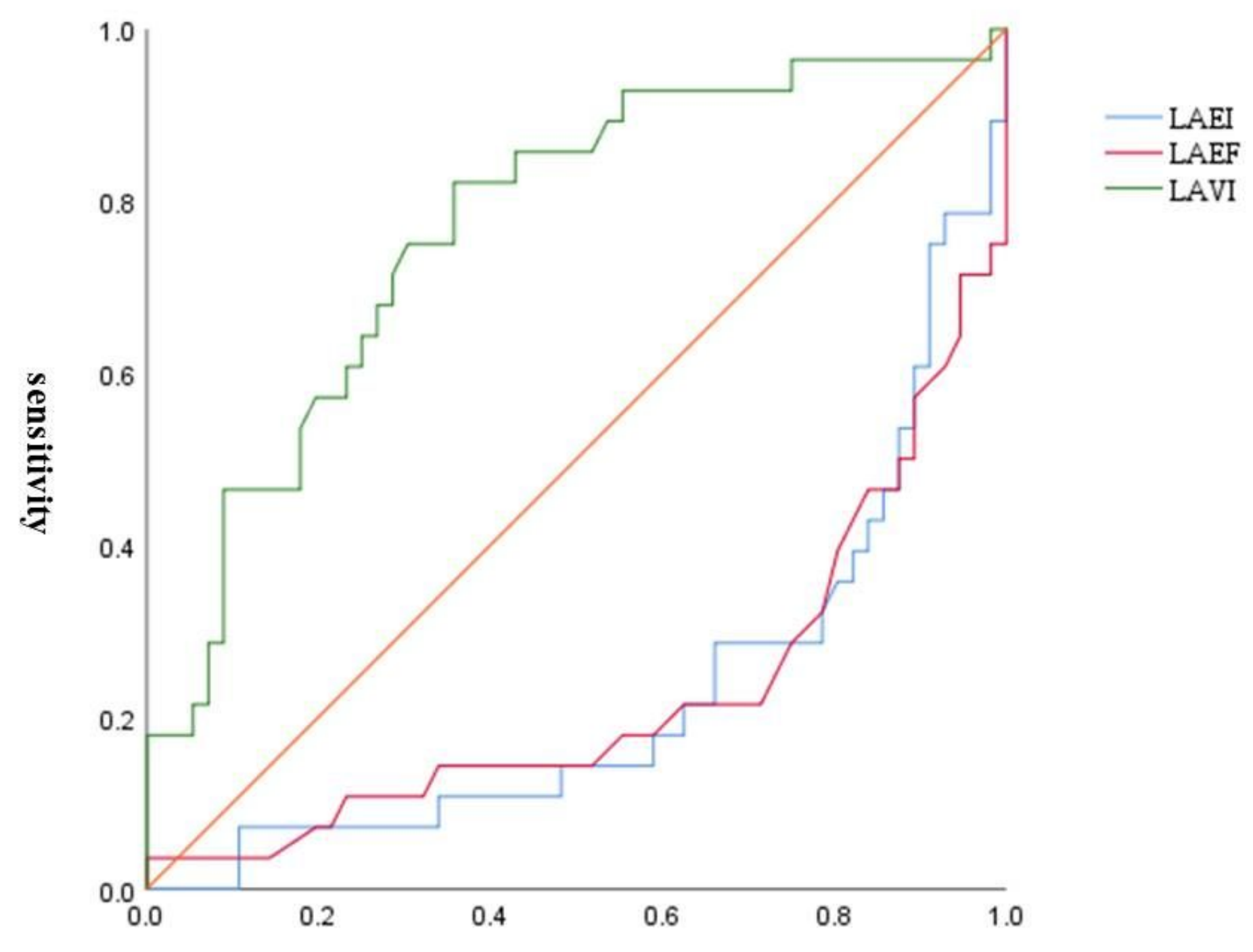

\section{1-specificity}

\section{Figure 1}

ROC curve for LAVI (cut-off value $\geq 24 \mathrm{ml} / \mathrm{m} 2$, AUC: 0.769, SE: 0.055, $p<0.001$ ), LA El (cut-off value $\leq 81$ $\%$, AUC: 0.224 , SE: $0.056, p<0.001$ ) and LA EF (cut-off value $\leq 56 \%$, AUC: 0.224 , SE: $0.058, p<0.001$ ). LAVI: left atrial volume index, LA El (LA expansion index), LA EF (LA ejection fraction). 

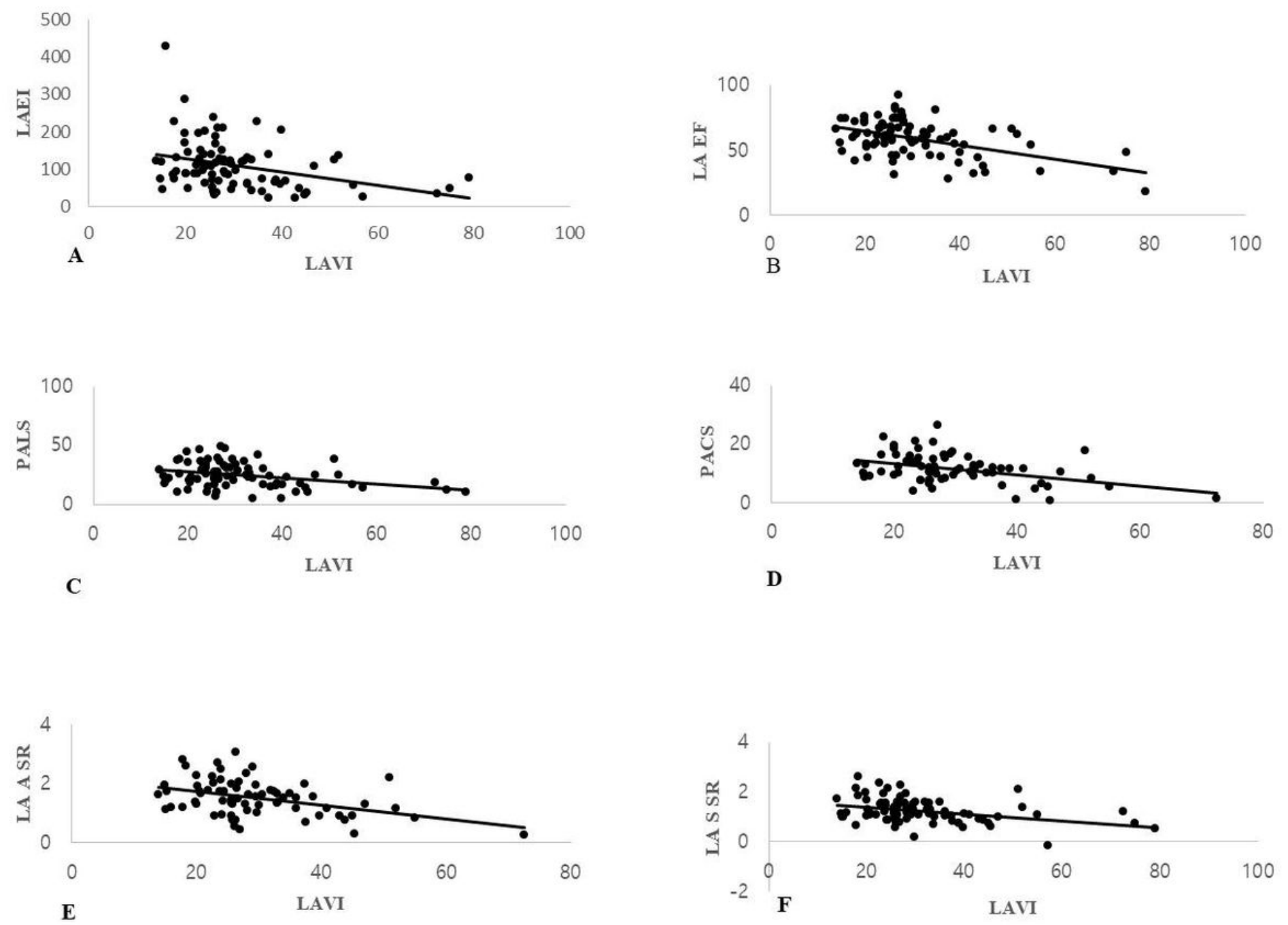

Figure 2

Inverse correlation of LA EI, EF, PALS, PACS, LA A SR and LA S SR with LAVI LAVI: left atrial volume index, LA El (LA expansion index), LA EF (LA ejection fraction), PALS (peak atrial longitudinal strain), PACS (peak atrial contraction strain), SR (strain rate)

\section{Supplementary Files}

This is a list of supplementary files associated with this preprint. Click to download.

- Centralimage.png 